\title{
Morphological changes in the spiracles of Anopheles gambiae s.l (Diptera) as a response to the dry season conditions in Burkina Faso (West Africa)
}

Wadaka Mamai ${ }^{1,2,3,7^{*}}$, Karine Mouline ${ }^{1,2}$, Jean-Philippe Parvy ${ }^{4,5}$, Jo Le Lannic ${ }^{6}$, Kounbobr Roch Dabiré1, Georges Anicet Ouédraogo ${ }^{3}$, David Renault ${ }^{6}$ and Frederic Simard ${ }^{2}$

\begin{abstract}
Background: Survival to dry season conditions of sub-Saharan savannahs is a major challenge for insects inhabiting such environments, especially regarding the desiccation threat they are exposed to. While extensive literature about insect seasonality has revealed morphologic, metabolic and physiological changes in many species, only a few studies have explored the responses following exposure to the stressful dry season conditions in major malaria vectors. Here, we explored morphological changes triggered by exposure to dry season conditions in An. gambiae s.l. mosquitoes by comparing females reared in climatic chambers reflecting environmental conditions found in mosquito habitats during the rainy and dry seasons in a savannah area of Burkina Faso (West Africa).

Results: Using scanning electron microscopy (SEM) and confocal imaging, we revealed significant changes in morphological features of the spiracles in females An. gambiae s.l. exposed to contrasted environmental conditions. Hence, the hairs surrounding the spiracles were thicker in the three species when raised under dry season environmental conditions. The thicker hairs were in some cases totally obstructing spiracular openings. Specific staining provided evidence against contamination by external microorganisms such as bacteria and fungi. However, only further analysis would unequivocally rule out the hypothesis of experimental artifact.

Conclusion: Morphological changes in spiracular features probably help to limit body water loss during desiccating conditions, therefore contributing to insect survival. Differences between species within the An. gambiae complex might therefore reflect different survival strategies used by these species to overcome the detrimental dry season conditions in the wild.
\end{abstract}

Keywords: Spiracle, Morphology, SEM, Desiccation, Anopheles gambiae, Burkina Faso

\section{Background}

Habitats of tropical savannahs are characterized by pronounced seasonal and daily fluctuations in environmental conditions such as temperature (with hot days and cool nights) and relative humidity. The conditions

\footnotetext{
* Correspondence: mwjosephfr@yahoo.fr

'Institut de Recherche en Sciences de la Santé (IRSS), Direction Régionale de I'Ouest (DRO), 399 Avenue de la Liberté, 01 BP 545 Bobo-Dioulasso, Burkina Faso

${ }^{2}$ MIVEGEC, UMR IRD 224-CNRS 5290-Université de Montpellier, Institut de Recherche pour le Développement, 911 Avenue Agropolis, BP 64501, 34394 Montpellier, cedex 5, France

Full list of author information is available at the end of the article
}

for malaria transmission in these regions are suitable only during certain periods of the year, particularly in the rainy season [1]. Thus, the vector dynamics, reproductive period and disease transmission intensity fluctuate considerably with this seasonal variation in temperature, precipitation and day length [2-4]. During the unfavourable (dry) weather, malaria mosquitoes of the Anopheles gambiae s.l. species complex are exposed to severe desiccation challenge, either through the drying-up of their breeding sites and/or the low ambient relative humidity [5-7]. Additionally, in early dry season conditions, mosquito larvae might further experience increased crowding 
while available surface water collections shrink and vanish. To survive through unfavourable conditions, many insects undergo dormancy (diapause or quiescence), characterized by a suite of morphological, physiological, biochemical and behavioural changes that enhance tolerance and extend survival to environmental stresses $[8,9]$ and in particular to desiccation [10].

The two molecular forms of An. gambiae s.s., recently named $A n$. coluzzii (former M molecular form) and $A n$. gambiae (former S molecular form) [11] and An. arabiensis are members of the Anopheles gambiae s.l. complex, a group of closely related and morphologically indistinguishable species [12]. Their distribution ranges encompass broad environmental and ecological settings, including arid and semi-arid areas. Although widely sympatric, the three species exhibit molecular, behavioural, physiological, and ecological differences [13-18]. Despite increased attention drawn in recent years on dry season survival strategies in these major African malaria vectors species [7, 19-21], little is known about the processes that sustains survival during the stressful dry season conditions. A recent field study showed evidence that aestivation (summer diapause) is one mechanism that allows $A n$. coluzzii to persist in the Sahel [7]. However, migration to/from more favourable localities where reproduction continues year-round might also be involved [19].

Suppression of water loss is a characteristic of species that face weather-induced desiccation [22]. It is also known that the main route of water loss is the cuticle, while water loss during respiration accounts for about $5-20 \%$ of the total water evaporation [23, 24]. Respiratory gas exchanges in mosquitoes occur through a multi-branched tracheal system, where cuticular openings called "spiracles" are located on the thorax and the abdomen. Spiracles are very variable structurally between genus and species, however, typically, the opening leads to a cavity (the atrium) from which the tracheae arise. In addition, spiracles of most insects have closing valves and can be surrounded by dust-catching hairs. In adult mosquitoes, spiracles are paired, bilaterally symmetric and located on the mesothorax, metathorax, and abdominal segments. Their apertures ensure the tradeoff between gas exchanges and water loss [7], since oxygen, a necessary gas for cell activity, must pass through the spiracles to enter the respiratory system [25]. Regulation of spiracle aperture plays a role in water conservation and may best be illustrated in insects showing discontinuous respiration $[26,27]$. Although the adaptive significance of discontinuous gas exchange (DGC) is a subject of considerable debate, this respiratory regimen is characterized by a period in which the spiracles are fully closed. Indeed, DGC is a repeating cycle of spiracular openings and closings that leads to periodic releases of carbon dioxide [28]. In ants Pogonomyrmex barbatus, the metabolic rates were found lowest for individuals using DGC, intermediate for individuals using cyclic gas exchange, and highest for individuals using continuous gas exchange [29]. Permanently opened spiracles allow maximum gas exchange but insects face desiccation stress more quickly [30]. Studying the effects of a xeric environment on water balance in Glossina sp., Bushel [31] concluded that increased water retention in Glossina sp from xeric environments resulted largely from spiracular control of transpiration. Spiracle size could also be positively correlated with water loss. Indeed, Nagpal and collaborators [32] showed that the spiracles of ecological variants of An. stephensi displayed different sizes, being the smallest in the xerophilic ecotype.

Water conservation mechanisms may be of considerable importance to survival and there is evidence that spiracles are instrumental in water conservation while still responding to the often-conflicting demands of respiration. The object of our study was therefore to survey, using electronic and confocal microscopy, the effect of contrasted environmental conditions on spiracles morphology of adult females of the An. gambiae s.l. complex raised under environmental conditions mimicking those found in a savannah area of Burkina Faso during the rainy and the dry seasons.

\section{Methods \\ Mosquitoes}

We used mosquito colonies maintained at the Institut de Recherche en Sciences de la Santé (IRSS) insectaries in Bobo-Dioulasso under controlled conditions $\left(27 \pm 1{ }^{\circ} \mathrm{C}\right.$, $80 \pm 10 \%$ relative humidity $(\mathrm{RH})$ and 12:12 dark/light cycle). The An. arabiensis colony originated from wild gravid females collected in Bobo-Dioulasso $\left(11^{\circ} 10^{\prime} \mathrm{N}, 4^{\circ}\right.$ $17^{\prime}$ W) in 2008, the $A n$. coluzzii colony was seeded from females collected in the village of Bama, Vallée du Kou $\left(11^{\circ} 23^{\prime} \mathrm{N}, 4^{\circ} 24^{\prime} \mathrm{W}\right)$ in 2008 , and the An. gambiae colony originated from females collected in the village of Soumousso $\left(11^{\circ} 04^{\prime} \mathrm{N}, 4^{\circ} 03^{\prime} \mathrm{W}\right.$ ) in 2009 (see [18] for further details). All these sites are located in southwestern Burkina Faso, within $50 \mathrm{~km}$ from each other and were previously described $[15,18,33]$.

\section{Environmental conditions, mosquito rearing and sample collection}

Mosquitoes were reared from the egg to the adult stage in programmable climatic chambers (Sanyo MLR 315H, Japan), where the climatic parameters characterizing the rainy and the dry season conditions were defined from hourly averaged records collected in Bama during August 2010 (rainy season, RS) (Additional file 1: Figure S1A) and December 2010 (dry season, DS) (Additional file 1: Figure S1C) using a Vantage Pro2 monitoring 
station (Weatherlink; Davis Instruments, Hayward, CA, U.S.A.) As previously described [18, 34], twelve steps cycles were designed to reproduce as close as possible the natural climatic variations monitored in the fields. A photoperiod of $12 \mathrm{~L}$ : 12D was set, corresponding to day length at the end of the rainy season in Bama (i.e., mid/ late October), when dry season survival strategies might be set up in sensitive stages. To control for potential cycle failure, temperature and relative humidity were recorded inside the chamber at a $10 \mathrm{~min}$ pace using MSR145 Data Loggers (MSR145B4HL, MSR electronics $\mathrm{GmBH}$, Switzerland). We also recorded the temperature cycles to which larvae were exposed using waterproof MSR145B4T2L monitors. The daily air temperature and humidity fluctuations recorded in the climatic chamber, identical to [18, 34], are represented in Additional file 1: Figure $\mathrm{S} 1 \mathrm{~B}$ and $\mathrm{D}$ and water temperature variation is shown in Additional file 2: Figure S2.

For each mosquito colony, eggs were collected from three independent batches, each produced by $>50$ caged females. Eggs were transferred into transparent plastic trays $(21.5 \times 16 \times 9.5 \mathrm{~cm})$ containing spring water and incubated in the climatic chambers. After hatching, firstinstars were counted and distributed into new plastic trays containing $1 \mathrm{~L}$ of spring water at a density of 100 larvae per tray. Each species was reared in separate pans, and three trays were used per colony and per environmental condition. The first two larval instars were fed every other day with $0.30 \mathrm{mg}$ of ground fish food (Tetra$\mathrm{min}^{\circ}$ ), whereas later instars were supplied with $0.75 \mathrm{mg}$ of food. Water was renewed when necessary to avoid scum formation and fouling of the media. The water used for renewal was stored in advance in the climatic chambers to avoid any perturbation of the temperature cycles. Every day, the position of the trays was randomly alternated to avoid positional effects within the incubators. Before adult emergence, pupae were collected with pipettes and transferred into plastic cups covered with netting. Emerging adults were immediately removed with an aspirator. Males were discarded and only females were kept in plastic cups closed with nets and fed using a cotton ball soaked with $10 \%$ glucose solution. Starting from the first day after their emergence, the dry season (DS) females (inside the cups) were placed into large plastic boxes filled with desiccant (Silica gel Chameleon(), where relative humidity reaches values as low as $15 \%$, as measured by a $\mathrm{T} / \mathrm{H}$ recorder MSR145 (MSR Electronics, GmBH, Switzerland). This was performed to mimic the field conditions, where relative humidity drops below levels allowed in the climatic chambers during the hottest hours in December (Additional file 1: Figure S1A). Hence, females were placed into the boxes (without access to sugar) at 10 a.m. and removed at 4 p.m., where a new cotton ball soaked with glucose solution was provided. For consistency, the same protocol was applied to the females raised under rainy season (RS) conditions, except that the boxes were filled with desiccant soaked with water. Environmental conditions monitored inside the boxes are provided in Additional file 1: Figure S1C and D. This was reproduced each day until the 10th day post emergence, where females of each species were collected around 2 p.m. at the very same time for each environmental condition to avoid potential confounding effect of circadian rhythms on spiracle aperture. Samples were snap frozen and preserved in $70 \%$ ethanol under ambient temperature. Twenty alcohol-preserved specimens were sent to the University of Rennes 1, France, before being prepared for further processing through scanning electron microscopy (below).

A second experiment involving An. coluzzii and $A n$. gambiae raised under the same dry season conditions as described above was further performed for confocal imaging (see below). This experiment involved 50 ethanol preserved mosquitoes from each species, which were sent to the University of Pierre et Marie Curie, Paris, France for spiracle staining and observations using confocal microscopy.

\section{Scanning Electron Microscopy (SEM)}

Eight females from the first experiment were randomly picked for each species and each environmental condition and processed for SEM observations. The females were dehydrated by immersion during $30 \mathrm{~min}$ in etha$\mathrm{nol} /$ water solutions of graded ethanol concentrations (70, 80, 90, 95 and $100 \%$ ), according to [35 ]. Mosquitoes were then incubated in acetone and kept until use. They were dried by the critical point method using liquid $\mathrm{CO}_{2}$ in a Balzers Critical Point Drier (Balzers Union FL-9496 Balzers/Furstentum Liechtenstein, Germany) apparatus attached to specimen holders and coated with gold and palladium in a sputter coater (FINE COAT ion sputter JFC-1100, JEOL, Japon). Finally, the specimens were observed under a scanning electron microscope (JEOL SJS-6301F, Japan).

\section{Confocal imaging and spiracle staining}

Females of the second experiment and those from the field (for which the species status was assessed using PCR technique [36]) were dedicated to the confocal imaging only, for which mosquito thoraces were dissected in Phosphate Buffer Saline (PBS) and fixed for $30 \mathrm{~min}$ in PBT (PBS + $0.1 \%$ Triton X-100) containing $4 \%$ of paraformaldehyde.

The thoraces of 25 randomly picked females from experiment 2 (seven from the species An. coluzzii, 18 from An. gambiae, see Table 1) were further stained with a mixture containing Phalloidin (a commonly used stain for F-actin filaments) and TO-PRO -3 (a commonly used stain for nucleic acids): after three washes of $10 \mathrm{~min}$ in 
Table 1 Number of opened and closed spiracles and number of spiracles displaying coated setae when observed under confocal microscope after staining with the vital stain TO-PRO-3. Unobservable spiracles are those for which the dissection and/or mounting steps before observation were unsuccessful. Observed mosquitoes were from experiment 2

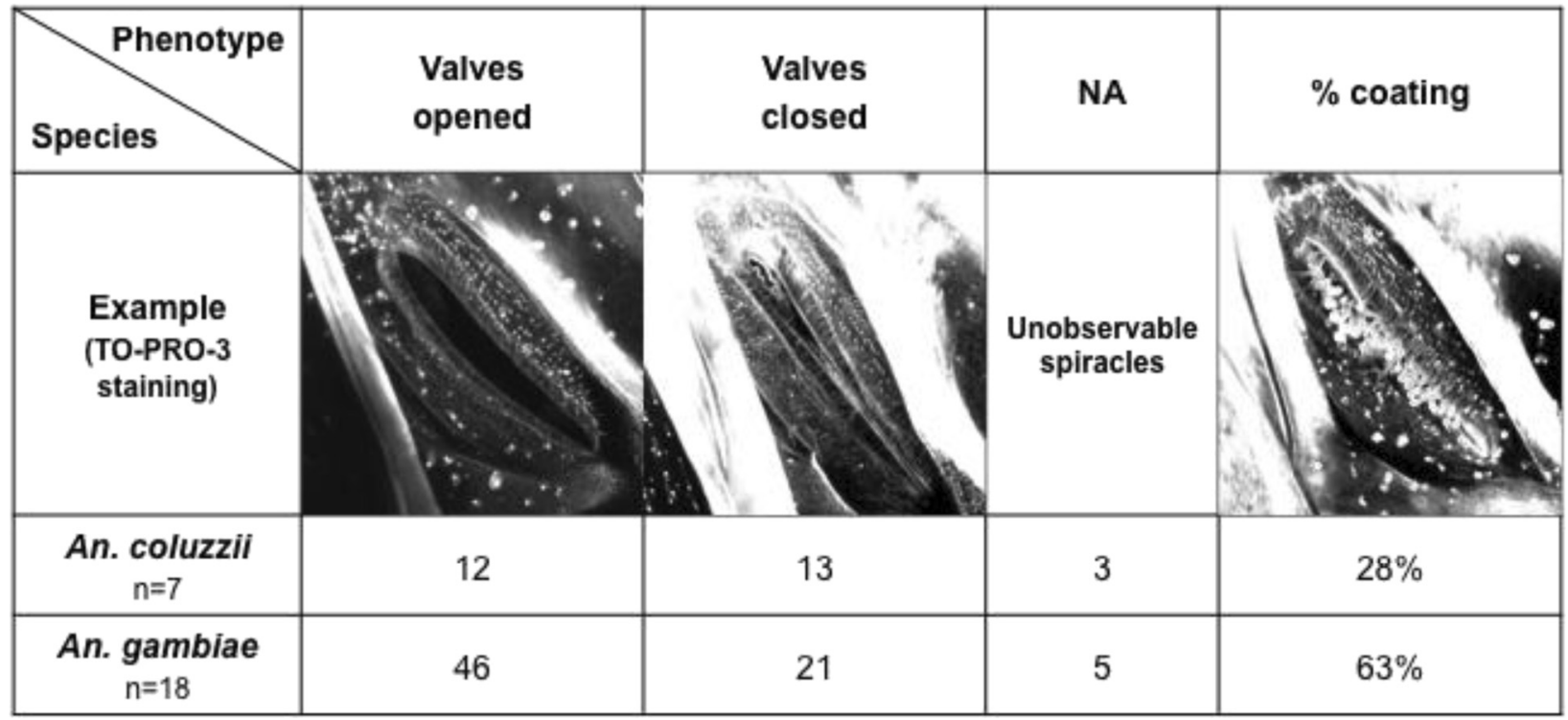

PBT, thoraces were stained overnight at $4{ }^{\circ} \mathrm{C}$ in $\mathrm{PBT}$ containing Alexa Fluor 568 Phalloidin $(0.07 \mu \mathrm{M})$ and TO-PRO ${ }^{\circ}-3$ (0.01 mM). Samples were rinsed 3 times for $10 \mathrm{~min}$ in PBT then hemi-thoraces were mounted with spiracles facing the coverslip in DABCO (Sigma) and examined by confocal microscopy using a Nikon (TE 2000-U) microscope.

\section{Results}

Morphological variation between species and environmental conditions

Entire females from experiment 1 were carefully observed under the SEM in order to look for striking morphological and structural differences between species and/or environmental conditions, with a focus on the respiratory system. The only differences that jumped out during our observations resided in the structural appearance of the spiracle apparatus.

The three species displayed differences in the visual aspect of the trichomes (or setae) of the mesothoracic spiracles in females reared in DS conditions compared to those reared in RS conditions (Fig. 1). Indeed, our observations showed that in $100 \%$ of the females observed under the SEM, the mesothoracic spiracles are wide open in females reared in RS conditions whereas the hairs appear wider and thicker in females reared under DS conditions, ultimately plugging entirely the spiracular aperture in An. coluzzii and An. arabiensis (Figs. 1 and 2). The phenotype is less striking in $A n$. gambiae females, where the hairs, although oversized, leave a wide aperture.
We were able to observe abdominal spiracles in only 4-5 females per species and conditions; however, all the spiracle structures observed in DS females were modified, and displayed obstructed apertures (Fig. 1). This suggests that the morphological modification of spiracular associated structures applies to the whole respiratory system. We confirmed our results in a second experiment where meso- and metathoracic spiracles of 25 females raised under DS conditions were observed under confocal imaging (Fig. 2, Table 1). Overall, about $63 \%$ of An. gambiae $(N=18)$ and $28 \%$ An. coluzzii $(N=7)$ females displayed oversized hairs around their thoracic spiracles under dry season conditions (Table 1). Thickened hairs were not always associated with the mechanical closure of the spiracular valves and both phenotypes can be independent although a significant trend appears. Of all the spiracle structures showing oversized hairs, $57 \% \quad$ (17 out of 30 ) were mechanically closed whereas this percentage was $28 \%$ (25 out 90 ) for the spiracles without coating $\left(\chi_{1}^{2}=\right.$ 7.033, $p=0.008)$.

Spiracle staining, observations, and related hypothesis on the origin of the oversized hairs

In all the mosquitoes we observed, neither Phalloidin (which stains F-actin filaments) nor TO-PRO ${ }^{\circ}-3$ (which stains nucleic acids) probes gave specific signals around or in the thickened hairs, whereas for $\mathrm{TO}-\mathrm{PRO}^{-}-3$, a specific staining was detected in the nuclei of mosquitoes' muscles (Fig. 3, asterisks). This staining is taken as our positive control and rules out a potential failure in the 

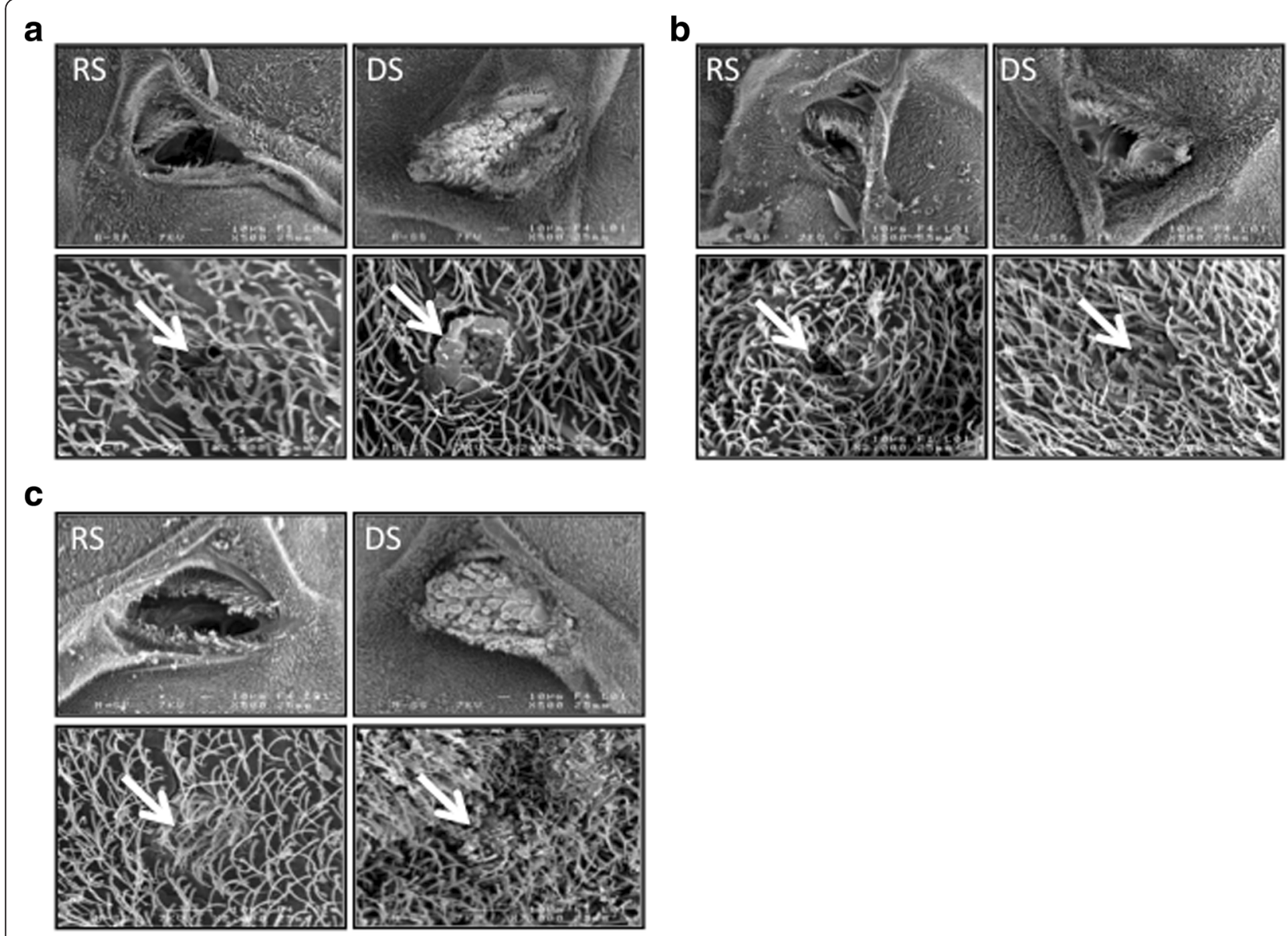

Fig. 1 Observation using SEM imaging of mesothoracic and abdominal spiracles of An. gambiae s.l. females raised under different environmental conditions (DS: Dry Season; RS: Rainy Season). Spiracle features surrounding spiracles are thickened under dry season conditions. White arrows point to the thickening of the spiracular features. a.: An. arabiensis; (b).: An. gambiae; (c).: An. coluzzii

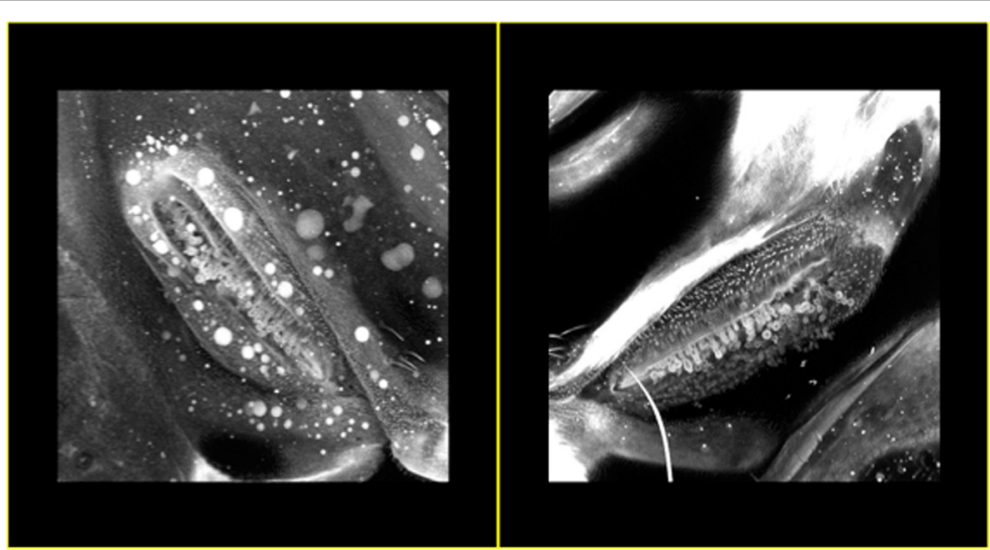

Fig. 2 Observation using confocal imaging showing a meta-thoracic spiracle with thickened hairs in two females An. coluzzii raised under dry season conditions 

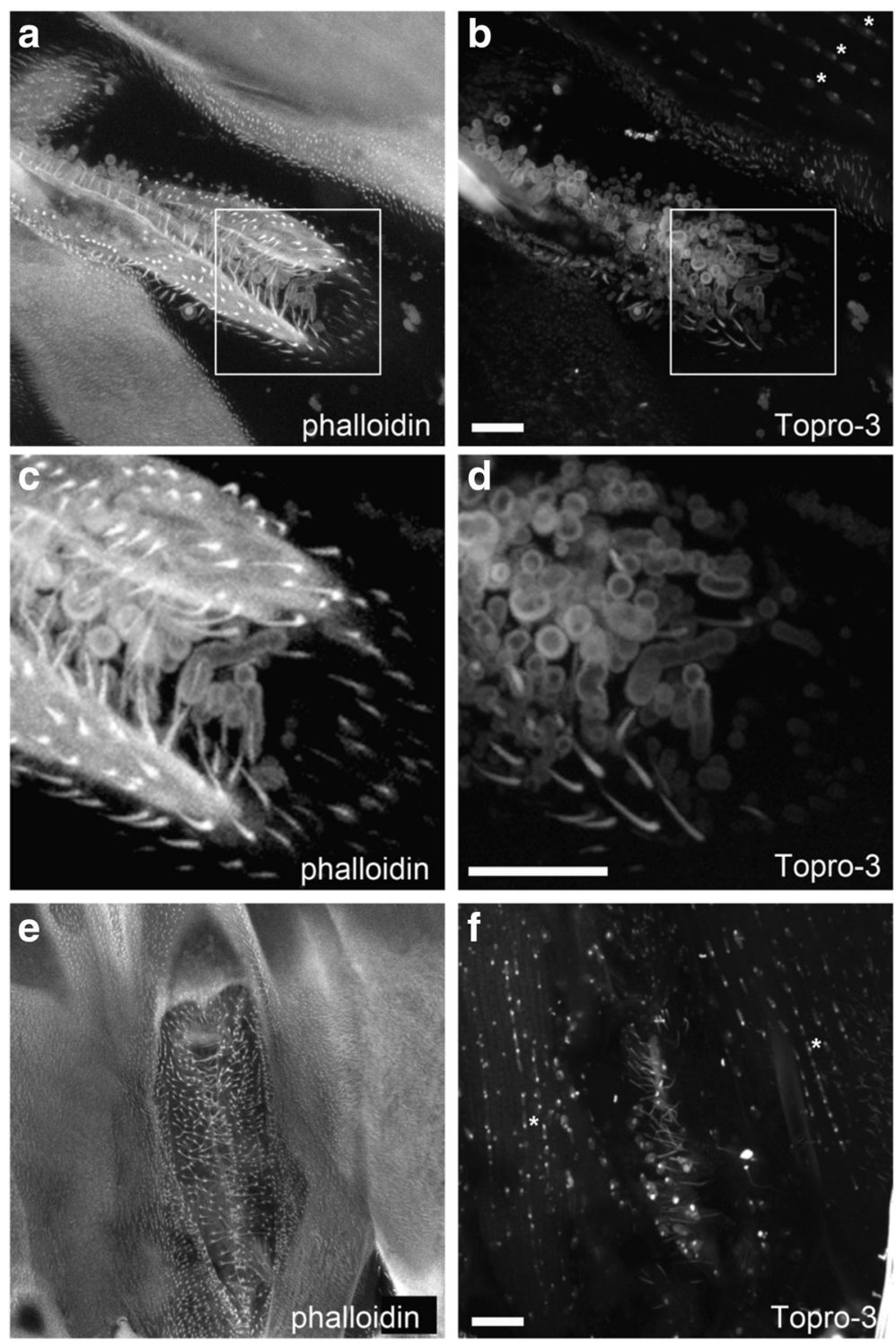

Fig. 3 Phalloidin (a, c and $\mathbf{e}$ ) and TO-PRO-3 (b, d and $\mathbf{f}$ ) staining of spiracles and thick setae in An. coluzzii females raised under dry season conditions. Spiracle structures were observed using confocal imaging

staining or detection procedures to explain the lack of signal in the setae.

Over 50 photographs under confocal imaging were taken that allowed the following observations: (i) in spiracles for which setae are not oversized, we can see that these structures are rooted in the insect's cuticle (Fig. 3e-f), (ii) setae are present at similar densities in all spiracles structures, whether they are oversized or not (Fig. 3f).

The lack of live cell specific staining, the observation that the oversized setae are differentiated from the mosquito cuticle and the fact that setae's densities between and specimen are equivalent among all the observed specimens, are all evidence against the hypothesis of this structure being built by the aggregation of contaminating microorganisms like bacteria or fungi. However, only further analysis of the qualitative nature of these structures would unequivocally rule out the hypothesis of experimental artifact.

\section{Discussion}

Despite the epidemiological importance of Anopheles mosquitoes, little is known about the mechanisms underpinning the survival of these species during the harsh conditions of the dry season in Africa. Here, we provide evidence for morphological variation in species of the An. gambiae s.l. complex in response to environmental variations. Because desiccation threat is more 
severe when spiracles are open [37, 38], we expected that mosquitoes reared in low ambient humidity and high temperatures, as typically observed during the dry season in sub-Saharan Africa, would exhibit morphological traits that serve as adaptations to reduce water loss. In fact, water is lost rapidly through opened spiracles during respiration $[12,39,40]$. In the arid-adapted ant Cataglyphis bicolor, the thoracic spiracles act as highcapacity gateways to the tracheal system and are responsible for approximately $90 \%$ of the total gas exchange including water vapour during running activity [41]. Moreover, studies from other insects have demonstrated that spiracle closure greatly facilitates water conservation [37, 42-45]. For example, by measuring the rate of water loss of tsetse flies in varying states of desiccation, Bursell [37] was able to show that spiracular control of transpiration increased as water reserves decreased.

The ability to close spiracles mechanically using the valve mechanism is a physiological adaptation that reduces water loss in insects [38, 46-48]. Hence, closed spiracles have been reported in Drosophila melanogaster during flight to reduce water loss and gas exchange into the tracheal system [38]. Although functional experiments are lacking to link the size or shape of the thoracic or abdominal spiracles with heat and/or desiccation tolerance, it is nonetheless clear that spiracular structures evolve towards smaller sizes in mosquito species inhabiting dry climates [49]. For instance, in the hygrophilous species of Anopheles and Aedes, spiracles are generally larger, whereas in the xerophilous species the openings are much smaller [30, 49]. Moreover, the spiracular index, i.e. the ratio of the length of the spiracle to the length of thorax has been used as a tool to identify ecological variants exposed to contrasted environments in $A n$. stephensi, this ratio being smaller in xeric vs mesic environments [32].

The mechanical closure of spiracles in desiccating conditions represents a rapid and transient response, which is thought to be regulated by a sensory mechanism elicited by low relative humidity [32]. In contrast, the specific spiracular structure modifications we observed through the whole respiratory system might represent important long-term adaptation, which could be programmed in anticipation to DS conditions. Hence, the ability to build oversized hairs that ultimately plug the spiracle apertures could be triggered by environmental cues sensed during the aquatic stages (i.e. high temperatures) or right upon emergence of adult mosquitoes (i.e. high temperatures and/or low relative humidity). Trichomes of various size, shape and density have been reported to line the spiracles atrium cavity in Geophilomorpha species, these structures being described as "solid and expended distally and showing a network of sclerotisation" in Cormocephalus calcaratus, "flap-like" in Strigamia, "cone shaped" in
Geophilus insculptus, or "elongated plates" in Hapiophilus subterraneous (Lewis, [50] and references therein). In his attempts to provide a functional explanation to such variability, Lewis [22], reported a strong correlation between the presence of a thick layer of trichomes in the atrium and resistance to desiccation challenge in 4 geophilomorphs. He concluded that this structure, together with a narrowed spiracular opening, might limit water evaporation from the atrium. Outgrowths cover the spiracular openings of the xerophilous buprestid beetle and, in the cockroach, such structures line the atrium, either outside or inside the valves (Hadley, [23] and references therein). Oversized setae around the spiracles may therefore well play the same role in mosquitoes than in the above-cited species, and might contribute to maintaining minimal metabolic activity while minimizing respiratory water loss. As such, they could be part of the survival strategies developed by An. gambiae s.l. to cope with low relative humidity values encountered during the dry season, either under diapause, quiescence or reproductively active states.

However, to our knowledge, our study is the first to report trichomes variability within the same species, putatively induced by environmental triggers. Dry season specific phenotype of the spiracles' setae was also observed in preliminary experiments involving mosquitoes collected in Bama at the beginning of the dry season. Two out of 22 mosquitoes observed under confocal imaging were showing oversized setae (i.e. $9 \%$ ), however, the phenotype was less striking in the sense that very few setae were indeed oversized (data not shown), which might suggest a progressive building of the phenotype over time, either with mosquito age and/or with the installation of the dry season. These preliminary observations warrant further investigations under field and/or semi-field conditions that will help resolve the biological meaning of such phenotypes as well as its underlying physiological and ecological determinants. Nonetheless, given that such structural variation has not been observed in any Anopheles mosquito to date, further experiments must also be conducted to strengthen our findings and to definitely rule out the caveat of an experimental artifact. Among these, a time course observation of the progressive building of oversized trichomes, careful observation of a recent study on Drosophila melanogaster revealed that lipid deposits are used to waterproof the spiracles [51], a link that might also exist in An. gambiae s.l. mosquitoes.

The ability to manage water reserves through the modification of spiracular morphology might account, at least in part, for better survival under desiccation challenge and increased body water content observed in mosquitoes when raised under dry season conditions [52]. In addition to water conservation, reduced spiracle openings might also contribute to lower metabolic rate 
and gas exchange through respiration. Huestis and collaborators [20] indicated that the mean metabolic rate of $A n$. coluzzii was lowest during the transition period between the wet and the dry season in the Sahel, which is consistent with obstructed spiracles as we observed in mosquitoes reared under DS conditions.

\section{Conclusion}

This study identified morphological variations in $A n$. gambiae s.l. mosquitoes when exposed to the severe dry season conditions in West African savannahs. These morphological changes might reflect specific adaptations to increase survival under different climatic or microclimatic conditions, pointing towards an important influence of spiracles's hairs on the rate of respiratory water loss and slowing down of the global metabolism. Although there is clear evidence for seasonal differentiation in $A n$. gambiae s.l. species, further research including cuticle morphology and composition and gas exchange rates are required to explore in more detail the biological relevance and adaptive value of these morphological adaptations.

\section{Additional files}

Additional file 1: Figure S1. Daily environmental conditions of temperature (dashed line), relative humidity (solid line), and dark/light duration (horizontal bars) recorded during the wet season in the field (A) inside the climatic chambers (B) and at the onset of the dry season in the field (C) and inside the climatic chambers (D). The red arrows indicate the times when females inside the cups were placed into large plastic boxes filled with desiccant (10 h) and were removed (18 h). Between the arrows, temperature and relative humidity values are those recorded inside the plastic boxes. Humidity values inside the climatic chamber are $2-5 \%$ above field ones for the rainy season conditions due to condensation problems in climatic chambers at higher humidity values. (TIF $870 \mathrm{~kb}$ )

Additional file 2: Figure S2. Daily temperature of the larval rearing water inside climatic chambers; wet season conditions (dashed line) and dry season conditions (solid line). (TIF $547 \mathrm{~kb}$ )

\section{Abbreviations}

DS: Dry Season; RS: Rainy Season; IRSS: Institut de Recherche en Sciences de la Santé; WHO: World Health Organisation; RH: Relative Humidity; SEM: Scanning Electron Microscopy.

\section{Competing interests}

The authors have declared that no competing interests exist.

\section{Authors' contributions}

KM, DR and FS, conceived the study and coordinated its implementation. RKD, GAO and JPP participated in the design. WM, KM and JPP performed the experiments and drafted the manuscript which was critically revised by DR, FS and RKD. All authors read and approved the final version of the manuscript.

\section{Acknowledgments}

This work is part of the project "Dry Season Survival Strategies in major African malaria vectors" funded by the French Agence Nationale de la Recherche through grant N ${ }^{\circ}$ ANR-08-MIEN-006 to F.S. WM is supported by a PhD fellowship from the IRD/DSF through "Bourse de soutien de thèse" program.

\section{Author details}

${ }^{1}$ Institut de Recherche en Sciences de la Santé (IRSS), Direction Régionale de I'Ouest (DRO), 399 Avenue de la Liberté, 01 BP 545 Bobo-Dioulasso, Burkina Faso. ${ }^{2}$ MIVEGEC, UMR IRD 224-CNRS 5290-Université de Montpellier, Institut de Recherche pour le Développement, 911 Avenue Agropolis, BP 64501, 34394 Montpellier, cedex 5, France. ${ }^{3}$ Université Polytechnique de Bobo-Dioulasso (UPB), Bobo-Dioulasso, Burkina Faso. ${ }^{4}$ Université Pierre et Marie Curie, 75005 Paris, France. ${ }^{5}$ CGM, UPR 3404, CNRS, 91190 Gif-sur-Yvette, France. ${ }^{6}$ Université de Rennes 1, UMR CNRS 6553 ECOBIO, Campus de Beaulieu, 263 Avenue du Gal Leclerc, CS 7420535042 Rennes, Cedex, France. ${ }^{7}$ Institut de Recherche pour le Développement, Antenne de Bobo Dioulasso, BP 171 Bobo Dioulasso 01, Burkina Faso.

Received: 1 December 2015 Accepted: 25 December 2015

Published online: 07 January 2016

\section{References}

1. Craig M, Snow R, LeSueur D. A climate-based distribution model of malaria transmission in sub-Saharan Africa. Trends Parasitol. 1999;15:105-11.

2. Ilboudo-Sanogo E, Tiono BA, Sagnon N, Cuzin ON, Nébié I, Sirima SB. Temporal dynamics of malaria transmission in two rural areas of Burkina Faso with two ecological differences. J Med Entomol. 2010;47(4):618-24.

3. Simard F, Lehmann T, Lemasson JJ, Diatta MD, Fontenille D. Persistence of Anopheles arabiensis during the severe dry season conditions in Senegal: an indirect approach using microsatellite loci. Insect Mol Biol. 2000;9:467-79.

4. Fontenille D, Lochouran L, Diagne N, Sokhna C, Lemasson JJ, Diatta M, et al. High annual and seasonal variations in malaria transmission by Anophelines and vector species composition in Dielmo, a holoendemic area in Senegal. Am J Trop Med Hyg. 1997;56(3):247-53.

5. Beier JC, Copeland R, Oyaro C, Masinya A, Odago WO, Oduor S, et al. Anopheles gambiae complex egg-stage survival in dry soil from larval development sites in western Kenya. J Am Mosq Control Assoc. 1990;6(1): $105-9$.

6. Koenraadt C, Paaijmans K, Githeko A, Knols B, Takken W. Egg hatching, larval movement and larval survival of the malaria vector Anopheles gambiae in desiccating habitats. Malar J. 2003;2:20.

7. Lehmann T, Dao A, Yaro AS, Adamou A, Kassogue Y, Diallo M, et al. Aestivation of the African malaria mosquito, Anopheles gambiae in the Sahel. Am J Trop Med Hyg. 2010;83:601-6.

8. Denlinger DL. Regulation of diapause. Annu Rev Entomol. 2002;47:93-122.

9. Kostál V, Denlinger DL. Dormancy and development in invertebrates. J Insect Physiol. 2011:57(5):537.

10. Gibbs A, Chippindale A, Rose M. Physiological mechanisms of evolved desiccation resistance in Drosophila melanogaster. J Exp Biol. 1997;200:1821-32.

11. Coetzee M, Hunt RH, Wilkerson R, Torre AD, Coulibaly MB, Besansky NJ. Anopheles coluzzii and Anopheles amharicus, new members of the Anopheles gambiae complex. Zootaxa. 2013;3619(3):246-74.

12. White G. Anopheles gambiae complex and disease transmission in Africa. Trans R Soc Trop Med Hyg. 1974;68:278-301.

13. Gimonneau G, Bouyer J, Morand S, Besansky NJ, Diabate A, Simard F. A behavioral mechanism underlying ecological divergence in the malaria mosquito Anopheles gambiae. Behav Ecol. 2010;21:1087-92.

14. Gimonneau G, Pombi M, Choisy M, Morand S, Dabire RK, Simard F. Larval habitat segregation between the molecular forms of the mosquito Anopheles gambiae in a rice field area of Burkina Faso, West Africa. Med Vet Entomol. 2012b;26:9-17.

15. Gimonneau G, Pombi M, Dabiré RK, Diabaté A, Morand S, Simard F. Behavioural responses of Anopheles gambiae sensu stricto $M$ and $S$ molecular form larvae to an aquatic predator in Burkina Faso. Parasit Vectors. 2012;5:65

16. Turner TL, Hahn MW, Nuzhdin SV. Genomic Islands of Speciation in Anopheles gambiae. PLoS Biol. 2005;3(9):e285.

17. Rund SSC, Lee SJ, Bush BR, Duffield GE. Strain- and sex-specific differences in daily flight activity and the circadian clock of Anopheles gambiae mosquitoes. J Insect Physiol. 2012;58:1609-19.

18. Mouline K, Mamai W, Agnew P, Tchonfienet M, Brengues C, Dabiré R, et al. Physiology and development of the M and S molecular forms of Anopheles gambiae in Burkina Faso (West Africa). Med Vet Entomol. 2012;26:447-54.

19. Adamou A, Dao A, Timbine S, Kassogue Y, Yaro AS, Diallo M, et al. The contribution of aestivating mosquitoes to the persistence of Anopheles gambiae in the Sahel. Malar J. 2011;10:151. 
20. Huestis DL, Yaro AS, Traoré Al, Dieter KL, Nwagbara Jl, Bowie AC, et al. Seasonal variation in metabolic rate, flight activity and body size of Anopheles gambiae in the Sahel. J Exp Biol. 2012;215:2013-21.

21. Huestis DL, Lehmann T. Ecophysiology of Anopheles gambiae s.l.: Persistence in the Sahel. Infect Genet Evol, 2014. doi: 10.1016/j.meegid.2014.05.027.

22. Lewis JGE. On the spiracle structure and resistance to dessiccation of four species of geophilomorph centipedes. Ent Exp \& Appl. 1963;6:89-94.

23. Hadley NF. Water Relations of Terrestrial Arthropods. San Diego, CA: Academic; 1994

24. Chown SL. Respiratory water loss in insects. Comp Biochem Physiol A. 2002; 133:791-804.

25. Sukontason KL, Methanitikorn R, Boonsriwong W, Piangjai S, Kurahashi $\mathrm{H}_{\text {, }}$ Vogtsberger RC, et al. Ultrastructure of spiracles of Musca domestica and Hydrotaea chalcogaster (Diptera: Muscidae). Parasitol Res. 2006;100:19-23.

26. Lighton JRB. Discontinuous gas exchange in insects. Annu Rev Entomol. 1996;41:309-24.

27. Sibul I, Kuusik A, Luik A, Voolma K. Influence of environmental conditions on the breathing rhythms of the pine weevil Hylobius abietis (Coleoptera: Curculionidae). Agron Res. 2006:4(1):63-77.

28. Gray M, Bradley TJ. Evidence from mosquitoes suggests that cyclic gas exchange and discontinuous gas exchange are two manifestations of a single respiratory pattern. J Exp Biol. 2006;209:1603-11.

29. Gibbs AG, Johnson RA. The role of discontinuous gas exchange in insects: the chthonic hypothesis does not hold water. J Exp Biol. 2004;207:3477-82.

30. Krafsur E. Behavior of thoracic spiracles of Aedes mosquitoes in controlled relative humidities. Ann Entomol Soc Am. 1971;64:93-7.

31. Bursell B. Tse-tse fly physiology. Bull World Health Organ. 1963;28:703-9.

32. Nagpal B, Srivastava A, Kalra N, Subbarao S. Spiracular indices in Anopheles stephensi: a taxonomic tool to identify ecological variants. J Med Entomol. 2003:40:747-9.

33. Dabiré RK, Namountougou M, Sawadogo SP, Yaro LB, Toé HK, Ouari A, et al. Population dynamics of Anopheles gambiae s.I. in Bobo-Dioulasso city: bionomics, infection rate and susceptibility to insecticides. Parasit Vectors. 2012;5:127.

34. Mamai W, Mouline K, Blais C, Larvor V, Dabiré KR, Ouedraogo GA, et al. Metabolomic and ecdysteroid Variations in Anopheles gambiae s.l. mosquitoes exposed to the stressful conditions of the dry season in Burkina Faso, West Africa. Physiol Biochem Zool. 2014;87(3):486-97.

35. Gothe R, Schöl H. Morphology and structural organization of spiracles in female Argas (Persicargas) walkerae (Acari: Argasidae). Exp Applied Acarol. 1992;14:151-63.

36. Santolamazza F, Mancini E, Simard F, Qi Y, Tu Z, della Torre A. Insertion polymorphisms of SINE200 retrotransposons within speciation islands of Anopheles gambiae molecular forms. Malar Journal. 2008;7:163.

37. Bursell B. Spiracular control of water loss in the tsetse fly. Proc Roy Entomol Soc London (A). 1957;32:21-9.

38. Lehman FO. Matching spiracle opening to metabolic need during flight in Drosophila. Science. 2001;294:1926-9.

39. Lighton JRB, Garrigan DA, Duncan FD, Johnson RA. Spiracular control of respiratory water loss in female alates of the harvester ant Pogonomyrmex rugosus. J Exp Biol. 1993;179:233-44.

40. Quinlan MC, Hadley NF. Gas exchange, ventilatory patterns, and water loss in two lubber grasshoppers: quantifying cuticular and respiratory transpiration. Physiol Zool. 1993;66:628-42.

41. Lighton JRB, Fukushi T, Wehner R. Ventilation in Cataglyphis bicolor: regulation of carbon dioxide release from the thoracic and abdominal spiracles. J Insect Physiol. 1993a;39:687-99.

42. Buck JB, Keister ML. Respiration and water loss in the adult blowfly, Phormia regina, and their relation to the physiological action of DDT. Biol Bull. 1949; 97:64-81.

43. Kanwisher JW. Tracheal gas dynamics in pupae of the cecropia silkworm. Biol Bull. 1966;130:96-105.

44. Mellanby K. The site of water loss from insects. Proc Roy Soc Ser B. 1934; 116:139-49.

45. Miller PL. Factors altering spiracle control in adult dragonflies: water balance. Exp Biol. 1964a;41:331-43.

46. Borrer DJ, Triplehorn CA, Johnson NF. An introduction to the study of insects. 6th ed. USA: Saunders College; 1989.

47. Kesler P. Respiration and respiratory water loss. In: Hoffmann K, editor. Environmental physiology and biochemistry of insects. Berlin Heidelberg New York: Springer; 1985. p. 137-84.
48. Richards OW, Davies RG. Imms' general textbook of entomology. $10^{\text {th }}$ edn, vol 1. Structure, physiology and development. London: Chapman and Hall; 1977.

49. Vinogradskaya ON, Buhorpa OH. The Spiracles of Anopheles and their Variations depending on the Xerophily and Hygrophily of the individual Species. Entomologicheskoe Obozrenie. 1950;31:151-4.

50. Lewis JGE. The biology of centipedes. Cambridge: Cambridge University Press; 1981.

51. Parvy JP, Napal L, Rubin T, Poidevin M, Perrin L, Wicker-Thomas C, et al. Drosophila melanogaster acetyl-coA carboxylase sustains a fatty aciddependent remote signal to waterproof the respiratory system. Plos Genet. 2012;8(8):e1002925.

52. Hidalgo K, Mouline K, Mamai W, Foucreau N, Dabiré KR, Bouchereau A, et al. Novel insights into the metabolic and biochemical underpinnings assisting dry-season survival in female malaria mosquitoes of the Anopheles gambiae complex. J Insect Physiol. 2014;70:102-16.

\section{Submit your next manuscript to BioMed Central and we will help you at every step:}

- We accept pre-submission inquiries

- Our selector tool helps you to find the most relevant journal

- We provide round the clock customer support

- Convenient online submission

- Thorough peer review

- Inclusion in PubMed and all major indexing services

- Maximum visibility for your research

Submit your manuscript at www.biomedcentral.com/submit
Biomed Central 